\title{
Tinkering with time
}

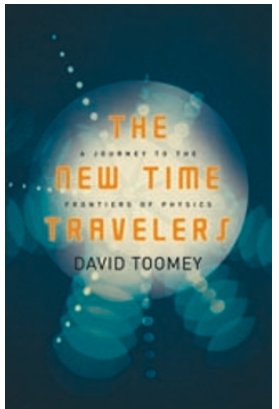

\section{THE NEW TIME TRAVELERS: A JOURNEY TO THE FRONTIERS OF PHYSICS BY DAVID TOOMEY}

W. W. Norton \& Co.: 2007. 320 pp. $\$ 25.95$

Years ago, David Toomey picked up H. G. Wells' The Time Machine and couldn't put it down. He was most interested in the drawing-room discussion between the time traveller and his friends in which time as a fourth dimension was discussed, but wanted to know more about just how that time machine might work. Toomey was therefore delighted to learn that that drawingroom conversation continues today - this time among physicists.

Toomey captures well the personalities of the 'new time travelers' - those physicists interested in whether time travel to the past is possible - from Stephen Hawking's sense of humour to Kip Thorne's penchant for placing scientific bets. Toomey has researched the history of the field extremely thoroughly. You will learn of British mathematician Charles Howard Hinton, who thought of world lines, which he called 'filamentary atoms', before Hermann Minkowski. You will learn that Carl Friedrich Gauss considered two-dimensional creatures, which he called 'bookworms' or 'flatworms', confined to live on an infinitely thin sheet of paper - well before Edwin Abbott's book Flatland.

A highlight of the book is its lucid discussion of time-travel paradoxes. What if you kill your grandmother as a young girl? Igor Novikov, Thorne and their colleagues favour the idea that the four-dimensional spacetime must be self consistent - time travellers never change the past, being always part of it: you can't kill your grandmother, because you didn't. Using extensive thought experiments with billiard balls going through wormholes, no matter how hard they tried to produce paradoxes, they could always find self-consistent solutions. David Deutsch has a different view. In the many-worlds theory of quantum mechanics, if you kill your grandmother in the past, you just make the universe branch off onto a parallel universe with a time traveller and a dead grandmother. The original parallel universe where the time traveller was born still exists.

When Wells wrote his book in 1895, the laws of physics (Newton's laws) allowed no time travel to either the future or the past. But in 1905, Albert Einstein's theory of special relativity showed moving clocks tick slowly, making time travel to the future possible. We now know a number of solutions to Einstein's equations of general relativity (1915) that are sufficiently twisted to allow time travel to the past: Kurt Gödel's 1949 rotating universe; the Morris-Thorne-Yurtsever wormhole (1988); the Tipler-van Stockum infinite rotating cylinder, moving cosmic strings (myself), the rotating black-hole interior (Brandon Carter), a Roman ring of wormholes (Matt Visser), the Everett-Alcubierre warp drive, my and Li-Xin Li's self-creating universe; Amos Ori's torus; and others. The book discusses all of these.

But can a time machine really be constructed? Hawking, like one of the time traveller's sceptical friends in Wells' drawing-room, put forward the chronology protection conjecture - that the laws of physics will somehow conspire to prevent time travel to the past. As Toomey describes, Hawking gathered a number of arguments. If a time machine is created where there was none before, there must be a Cauchy horizon separating the no-time-travel and time-travel regions and the quantum vacuum might blow up as one approached the Cauchy horizon. Secondly, if the time machine is made of ordinary positive-energydensity stuff, the solution is unstable approaching the Cauchy horizon and singularities may occur.

Yet, there are loopholes. Alternative quantum vacuum states can be found in some cases that do not blow up at the Cauchy horizon, and wormholes avoid instability by using exotic matter (the Casimir vacuum). Alternatively, stable singularity-free solutions can be found if the time loop occurs at the very beginning of the universe. To understand whether it is possible to construct any of these time machines, the author rightly concludes we may need to understand the laws of quantum gravity - how gravity behaves on microscopic scales - one reason physicists working on this problem find it so fascinating.

\section{J. Richard Gott}

J. R. Gott is in the Department of Astrophysical Sciences, Princeton University, Princeton, New Jersey 08544, USA, and is the author of Time Travel in Einstein's Universe (Mariner Books, 2002).

\section{On our bookshelf}

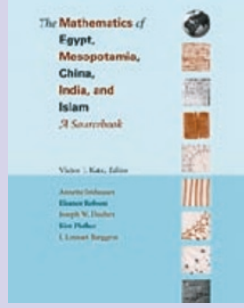

The Mathematics of Egypt, Mesopotamia, China, India, and Islam: A Sourcebook edited by Victor J. Katz Princeton Univ. Press: 2007. 685 pp. $\$ 75$

We're aware that the ancient cultures were mathematically advanced. Now translations of early texts from five key regions are available together for the first time, and put into context by experts.

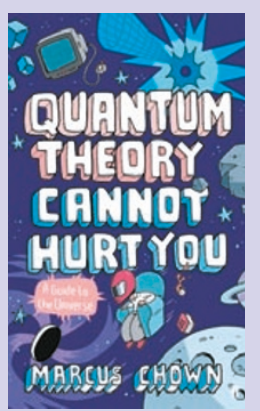

Quantum Theory Cannot

Hurt You: A Guide to the Universe by Marcus Chown

Faber and Faber: 2007. 200 pp. $£ 12.99$

Quantum theory and Einstein's general theory of relativity made simple? To be read in one morning? Chown shares his amazement at "how much stranger science is than science fiction". 